\begin{tabular}{ccc}
\hline Jif & Journal of Mathematical Analysis and Modeling \\
jmam.sabapub.com & J Math Anal \& Model \\
ISSN 2709-5924 & (2020)1(1): 87-98 \\
& & doi:10.48185/jmam.v1i1.41 \\
\hline
\end{tabular}

\title{
Certain investigations in the field of special functions
}

\author{
Maisoon A. Kulib ${ }^{a}$, Ahmed A. Al-Gonah ${ }^{b, *}(\mathbb{0})$, Salem S. Barahmah ${ }^{c}$
}

aDepartment of Basic Science, Faculty of Engineering, Aden University, Aden, Yemen. b2Department of Mathematics, Faculty of Science, Aden University, Aden, Yemen.

${ }^{c}$ Department Mathematics, Faculty of Education, Aden University, Aden, Yemen.

\begin{abstract}
Motivated mainly by a variety of applications of Euler's Beta, hypergeometric, and confluent hypergeometric functions together with their extensions in a wide range of research fields such as engineering, chemical, and physical problems. In this paper, we introduce modified forms of some extended special functions such as Gamma function, Beta function, hypergeometric function and confluent hypergeometric function by making use of the idea given in reference [1]. Also, certain investigations including summation formulas, integral representations and Mellin transform of these modified functions are derived. Further, many known results are obtained as special cases of our main results.
\end{abstract}

Keywords: Gamma function, Beta function, Hypergeometric function.

2010 MSC: 11S80, 33C05.

\section{Introduction}

The classical Gamma and Beta functions are defined respectively as follows (see [2]):

$$
\Gamma(x)=\int_{0}^{\infty} t^{x-1} e^{-t} d t, \quad(\operatorname{Re}(x)>0) .
$$

and

$$
B(x, y)=\int_{0}^{1} t^{x-1}(1-t)^{y-1} d t, \quad(\operatorname{Re}(x)>0, \operatorname{Re}(y)>0),
$$

for each $x, y \in(0,+\infty)$. The Gamma and Beta functions posses the following relation:

$$
B(x, y)=\frac{\Gamma(x) \Gamma(y)}{\Gamma(x+y)} .
$$


Recently, many authors have introduced certain extensions for Gamma and Beta functions (see $[3,4,5,6,7,8,9,10]$ ) and studied its properties. Chaudhry and Zubair [11] introduced the following extended Gamma function:

$$
\Gamma_{p}(x)=\int_{0}^{\infty} t^{x-1} \exp \left(-t-\frac{p}{t}\right) d t, \quad(\operatorname{Re}(x)>0, \operatorname{Re}(p) \geqslant 0) .
$$

Chaudhry et al., [3] presented the following extended Beta function:

$$
\begin{gathered}
B(x, y ; p)=\int_{0}^{1} t^{x-1}(1-t)^{y-1} \exp \left(\frac{-p}{t(1-t)}\right) d t, \\
(\operatorname{Re}(x)>0, \operatorname{Re}(y)>0, \operatorname{Re}(p) \geqslant 0) .
\end{gathered}
$$

Note that, for $p=0,(1.4)$ and (1.5) reduce to (1.1) and (1.2).

Also, the extended hypergeometric and confluent hypergeometric functions are introduced in [4] as follows:

$$
F_{p}(a, b ; c ; z)=\sum_{n=0}^{\infty} \frac{B_{p}(b+n, c-b)}{B(b, c-b)}(a)_{n} \frac{z^{n}}{n !},
$$

where $\operatorname{Re}(p) \geqslant 0$ and $\operatorname{Re}(\mathrm{c})>\operatorname{Re}(\mathrm{b})>0,|z|<1$.

$$
\phi_{p}(b ; c ; z)=\sum_{n=0}^{\infty} \frac{B_{p}(b+n, c-b)}{B(b, c-b)} \frac{z^{n}}{n !},
$$

where $\operatorname{Re}(p) \geqslant 0$ and $\operatorname{Re}(\mathrm{c})>\operatorname{Re}(b)>0$.

It is clear that when $p=0$, the equations (1.6) and (1.7) reduce to the well known classical hypergeometric and confluent hypergeometric functions respectively.

Very recently, the modified Laplace transform is introduced by Saif et al. [1] as follows:

$$
\mathrm{L}_{\mathbf{a}}\{\mathrm{f}(\mathrm{t})\}=\int_{0}^{\infty} \mathrm{a}^{-\mathrm{st}} \mathrm{f}(\mathrm{t}) \mathrm{dt}, \quad \operatorname{Re}(\mathrm{s})>0, \mathrm{a} \in(0, \infty) \backslash\{1\},
$$

which for $a=e$ reduces to the known Laplace transform given as:

$$
L\{f(t)\}=\int_{0}^{\infty} e^{-s t} f(t) d t, \quad \operatorname{Re}(s)>0,
$$

Motivated by the idea given in [1], in this paper, we introduce a new modified forms of the extended Gamma, Beta, hypergeometric and confluent hypergeometric functions defined in equations (1.4)-(1.7) respectively.

\section{Modified Gamma and Beta functions}

In this section, we introduce a new modified forms of extended Gamma and Beta functions in the following forms :

$$
\Gamma_{p}(x ; a)=\int_{0}^{\infty} t^{x-1} a^{\left(-t-\frac{p}{t}\right)} d t,
$$


for $\operatorname{Re}(x)>0, \operatorname{Re}(p)>0 a \in(0, \infty) \backslash\{1\}$, and

$$
B_{p}(x, y ; a)=\int_{0}^{1} t^{x-1}(1-t)^{y-1} a^{\left(\frac{-p}{t(1-t)}\right)} d t,
$$

for $\operatorname{Re}(x)>0, \operatorname{Re}(y)>0, \operatorname{Re}(p)>0$, and $a \in(0, \infty) \backslash\{1\}$.

Remark 2.1. For $p=0$, definition (2.1) reduces to a new modified Gamma function defined as:

$$
\Gamma(x ; a)=\int_{0}^{\infty} t^{x-1} a^{-t} d t, \quad \operatorname{Re}(x)>0, \text { and } a \in(0, \infty) \backslash\{1\},
$$

which for $x=n+1, n=0,1,2, \ldots$ gives the following relation between $\Gamma(x ; a)$ and the recently known result [[1], p. 129] (for $s=1$ ) :

$$
\Gamma(n+1 ; a)=\int_{0}^{\infty} t^{n} a^{-t} d t=\frac{\Gamma(n+1)}{(\log a)^{n+1}}=\frac{n !}{(\log a)^{n+1}} .
$$

Also, we note that

$$
\begin{gathered}
\Gamma_{p}(x ; e)=\Gamma_{p}(x), \\
\Gamma_{0}(x ; e)=\Gamma(x ; e)=\Gamma(x), \\
B_{p}(x, y ; e)=B_{p}(x, y), \\
B_{0}(x, y ; e)=B(x, y) .
\end{gathered}
$$

Now, we introduce certain properties of the modified forms of the extended Gamma and Beta functions in the form of the following theorems:

Theorem 2.2. The following summation formulas for $\Gamma_{p}(x ; a)$ and $B_{p}(x, y ; a)$ hold true:

$$
\begin{aligned}
\Gamma_{p}(x ; a) & =\sum_{n=0}^{\infty} \frac{(-p)^{n}(\log a)^{n}}{n !} \Gamma(x-n ; a), \\
B_{p}(x, y ; a) & =\sum_{n=0}^{\infty} \frac{(-p)^{n}(\log a)^{n}}{n !} B(x-n, y-n) .
\end{aligned}
$$

Proof. To prove (2.8), expanding the R.H.S. of equation (2.1) by using the following relation:

$$
a^{x}=\sum_{n=0}^{\infty} \frac{x^{n}(\log a)^{n}}{n !}
$$

we get

$$
\Gamma_{p}(x ; a)=\int_{0}^{\infty} t^{x-1} a^{-t} \sum_{n=0}^{\infty}\left(\frac{-p}{t}\right)^{n} \frac{(\log a)^{n}}{n !} d t,
$$

which on interchanging the order of summation and integration and then using relation (2.1) yields the desired result (2.8).

Similarly, proceeding on the same lines of proof (2.8) and using relation (1.2), we get result (2.9) and thus the proof of Theorem 2.1 is completed. 
Remark 2.3. Using the relation (1.3) in the R.H.S. of relation (2.9) and then applying some properties of pochhammer symbol, we get the following hypergeometric representation for $B_{p}(x, y ; a)$ :

$$
B_{p}(x, y ; a)=B(x, y){ }_{2} F_{2}\left(\frac{1-x-y}{2}, \frac{2-x-y}{2} ; 1-x, 1-y ; 4 p \log a\right),
$$

where ${ }_{r} F_{s}(\cdot)$ is the generalized hypergeometric function (see [12] or [2]).

Theorem 2.4. The following summation formulas for $\mathrm{B}_{\mathrm{p}}(x, y ; a)$ hold true:

$$
\begin{gathered}
B_{p}(x, 1-y ; a)=\sum_{n=0}^{\infty} \frac{(y)_{n}}{n !} B_{p}(x+n, 1 ; a), \\
B_{p}(x, y ; a)=\sum_{n=0}^{\infty} B_{p}(x+n, y+1 ; a), \\
B_{p}(x, y ; a)=\sum_{k=0}^{n}\left(\begin{array}{l}
n \\
k
\end{array}\right) B_{p}(x+k, y+n-k ; a), n \in N_{0} .
\end{gathered}
$$

Proof. To prove result (2.12). From (2.2), we have

$$
B_{p}(x, 1-y ; a)=\int_{0}^{1} t^{x-1}(1-t)^{-y} a^{\left(\frac{-p}{t(1-t)}\right)} d t .
$$

Using the generalized binomial theorem

$$
(1-t)^{-y}=\sum_{n=0}^{\infty} \frac{(y)_{n}}{n !} t^{n}, \quad|t|<1,
$$

we obtain

$$
B_{p}(x, 1-y ; a)=\int_{0}^{1} \sum_{n=0}^{\infty} \frac{(y) n}{n !} t^{x+n-1} a^{\left(\frac{-p}{t(1-t)}\right)} d t .
$$

Now, interchanging the order of summation and integration in the above equation and using (2.2), we get the required result (2.12). The proof of result (2.13) is similar to that of (2.12).

To prove equation (2.14), we use the mathematical induction on $\left(n \in N_{0}\right)$ as follows:

Clearly, For $n=0$ the equation (2.14) holds.

For $n=1$, we have

$$
\begin{aligned}
B_{p}(x+1, y ; a)+B_{p}(x, y+1 ; a) & =\int_{0}^{1}\left\{t^{x}(1-t)^{y-1}+t^{x-1}(1-t)^{y}\right\} a^{\left(\frac{-p}{t(1-t)}\right)} d t \\
& =\int_{0}^{1} t^{x-1}(1-t)^{y-1}\{t+(1-t)\} a^{\left(\frac{-p}{t(1-t)}\right)} d t \\
& =\int_{0}^{1} t^{x-1}(1-t)^{y-1} a^{\left(\frac{-p}{t(1-t)}\right)} d t \\
& =B_{p}(x, y ; a) .
\end{aligned}
$$


Therefore, the equation (2.14) holds for $n=1$.

Continuing this process for all $\left(n \in N_{0}\right.$ ), we finally obtain the desired relation (2.14) and thus the proof of Theorem 2.4 is completed.

Theorem 2.5. The following integral formulas for $\Gamma_{p}(x ; a)$ and $B_{p}(x, y ; a)$ hold true:

$$
\begin{gathered}
\int_{0}^{\infty} \Gamma_{p}(x ; a) p^{s-1} \mathrm{dp}=\Gamma(s ; a) \Gamma(x+s ; a),(\operatorname{Re}(s)>0, \operatorname{Re}(x+s)>0), \\
\int_{0}^{\infty} B_{p}(x, y ; a) p^{s-1} \mathrm{dp}=\Gamma(s ; a) B(x+s, y+s) \\
(\operatorname{Re}(s)>0, \operatorname{Re}(x+s)>0, \operatorname{Re}(y+s)>0) .
\end{gathered}
$$

Proof. Multiplying (2.1) by $p^{s-1}$ and integrating with respect to $p$ from $p=0$ to $p=\infty$, we get

$$
\int_{0}^{\infty} \Gamma_{p}(x ; a) p^{s-1} d p=\int_{0}^{\infty} p^{s-1} \int_{0}^{1} t^{x-1} a^{-t-\frac{p}{t}} d p d t
$$

The order of integration in (2.18) can be interchanged because of the uniform convergence of the integral. Therefore, we have

$$
\int_{0}^{\infty} \Gamma_{p}(x ; a) p^{s-1} d p=\int_{0}^{1} t^{x-1} a^{-t}\left(\int_{0}^{\infty} p^{s-1} a^{-\frac{p}{t}} d p\right) d t,
$$

which on putting $u=\frac{p}{t}$ and using relation (2.1) yields the desired result (2.16).

Similarly, proceeding on the same lines of proof (2.16) and using relation (2.1) and (1.2), we get the desired result (2.17) and thus the proof of Theorem 2.5 is completed.

Remark 2.6. Putting $s=1$ in relations (2.16) and (2.17), we get the following results:

Corollary 2.7. The following integral formulas for $\Gamma_{p}(x ; a)$ and $B_{p}(x, y ; a)$ hold true:

$$
\begin{aligned}
\int_{0}^{\infty} \Gamma_{p}(x ; a) d p & =\frac{1}{\log a} \Gamma(x+1 ; a), \\
\int_{0}^{\infty} B_{p}(x, y ; a) d p & =\frac{1}{\log a} B(1+x, 1+y) .
\end{aligned}
$$

Theorem 2.8. The following integral representations hold true:

$$
\begin{gathered}
B_{p}(x, y ; a)=2 \int_{0}^{\frac{\pi}{2}} \cos ^{2 x-1} \theta \sin ^{2 y-1} \theta a^{-p\left(\frac{1}{(\cos \theta \sin \theta)^{2}}\right)} d \theta, \\
B_{p}(x, y ; a)=\int_{0}^{\infty} \frac{u^{x-1}}{(1+u)^{x+y}} a^{-p\left(\frac{(1+u)^{2}}{u}\right)} d u, \\
B_{p}(x, y ; a)=(c-a)^{1-x-y} \int_{a}^{c}(u-a)^{x-1}(c-u)^{y-1} a^{-p\left(\frac{(c-a)^{2}}{(u-a)(c-u)}\right)} d u .
\end{gathered}
$$


Proof. To prove result (2.22), putting $t=\cos ^{2} \theta$ in (2.2), we have

$$
B_{p}(x, y ; a)=2 \int_{0}^{\frac{\pi}{2}} \cos ^{2 x-2} \theta \sin ^{2 y-2} \theta a^{-p\left(\frac{1}{\cos ^{2} \theta \sin ^{2} \theta}\right)} \cos \theta \sin \theta d \theta
$$

which yields the desired result (2.22).

Similarly, results (2.23) and (2.24) can be proved by taking the transformations $t=$ $\frac{u}{1+u}$ and $t=\frac{\mathfrak{u}-a}{c-a}$ in (2.2) respectively and thus the proof of Theorem 2.8 is completed.

Theorem 2.9. For the product of two modified gamma function $\Gamma_{\mathrm{p}}(\mathrm{x} ; \mathrm{a})$, the following formula holds true:

$$
\Gamma_{p}(x ; a) \Gamma_{p}(y ; a)=2 \int_{0}^{\infty}(r)^{2(x+y)-1} a^{-r^{2}} B \frac{p}{r^{2}}(x, y ; a) d r, \operatorname{Re}(x), \operatorname{Re}(y)>0 .
$$

Proof. Substituting $t=\eta^{2}$ in (2.1), we get

$$
\Gamma_{p}(x ; a)=2 \int_{0}^{\infty} \eta^{2 x-1} a^{\left(-\eta^{2}-\frac{p}{\eta^{2}}\right)} d \eta .
$$

Therefore,

$$
\begin{aligned}
\Gamma_{p}(x ; a) \Gamma_{p}(y ; a) & =4 \int_{0}^{\infty} \int_{0}^{\infty} \eta^{2 x-1} \rho^{2 y-1} a^{\left(-\eta^{2}-\frac{p}{\eta^{2}}\right)} a^{\left(-\rho^{2}-\frac{p}{\rho^{2}}\right)} d \eta d \rho \\
& =4 \int_{0}^{\infty} \int_{0}^{\infty} \eta^{2 x-1} \rho^{2 y-1} a^{-\left(\eta^{2}+\rho^{2}\right)} a^{-p\left(\frac{1}{\eta^{2}}+\frac{1}{\rho^{2}}\right)} d \eta d \rho \\
& =4 \int_{0}^{\infty} \int_{0}^{\infty} \eta^{2 x-1} \rho^{2 y-1} a^{-\left(\eta^{2}+\rho^{2}\right)} a^{-p\left(\frac{\rho^{2}+}{\eta^{2}} \frac{\eta^{2}}{\rho^{2}}\right)} d \eta d \rho .
\end{aligned}
$$

Substituting $\eta=r \cos \theta, \rho=r \sin \theta$, we get

$$
\begin{aligned}
& \Gamma_{p}(x ; a) \Gamma_{q}(y ; a) \\
= & 4 \int_{0}^{\frac{\pi}{2}} \int_{0}^{\infty}(r)^{2(x+y)-1}(\cos \theta)^{2 x-1}(\sin \theta)^{2 y-1} a^{-r^{2}} a^{-\frac{p}{r^{2}}\left(\frac{1}{(\cos \theta \sin \theta)^{2}}\right)} d r d \theta \\
= & 2 \int_{0}^{\infty}(r)^{2(x+y)-1} a^{-r^{2}}\left(2 \int_{0}^{\frac{\pi}{2}}(\cos \theta)^{2 x-1}(\sin \theta)^{2 y-1} a^{-\frac{p}{r^{2}}\left(\frac{1}{(\cos \theta \sin \theta)^{2}}\right)} d \theta\right) d r,
\end{aligned}
$$

which on using relation (2.22) yields the desired result (2.25).

Remark 2.10. (i) For $a=e$ and using relations (2.4) and (2.5), all the above relations related to $\Gamma_{p}(x ; a)$ reduce to the known results due to Chaudhry and Zubair [11].

(ii) For $a=e$ and using relation (2.6), all the above relations related to $B_{p}(x, y ; a)$ reduce to the known results due to Chaudhry et al. [3]. 


\section{Modified hypergeometric functions}

In this section, we introduce modified forms for the extension of hypergeometric and confluent hypergeometric functions given in (1.6) and (2.8) by using the modified Beta function defined in (2.2).

Definition 3.1. The modified forms of the extension of hypergeometric and confluent hypergeometric functions are defined as

$$
F_{p}(\alpha, \beta ; \gamma ; z ; a)=\sum_{n=0}^{\infty}(\alpha)_{n} \frac{B_{p}(\beta+n, \gamma-\beta ; a)}{B(\beta, \gamma-\beta)} \frac{z^{n}}{n !},
$$

for $\operatorname{Re}(\gamma)>\operatorname{Re}(\beta)>0, \operatorname{Re}(p) \geqslant 0, a \in(0, \infty) \backslash\{1\}$, and $|z|<1$.

$$
\phi_{p}(\beta ; \gamma ; z ; a)=\sum_{n=0}^{\infty} \frac{B_{p}(\beta+n, \gamma-\beta ; a)}{B(\beta, \gamma-\beta)} \frac{z^{n}}{n !},
$$

for $\operatorname{Re}(\gamma)>\operatorname{Re}(\beta)>0, \operatorname{Re}(p) \geqslant 0, a \in(0, \infty) \backslash\{1\}$, and $|z|<1$.

Remark 3.2. It is clear that, for $a=e,(3.1)$ and (3.2) reduce to the extended hypergeometric and confluent hypergeometric functions (1.6) and (1.7) respectively.

Now, we prove some properties of the modified hypergeometric and confluent hypergeometric functions $F_{p}(\alpha, \beta ; \gamma ; z ; a)$ and $\phi_{p}(\beta ; \gamma ; z ; a)$ in the form of the following theorems:

Theorem 3.3. The modified hypergeometric and confluent hypergeometric functions

$$
F_{p}(\alpha, \beta ; \gamma ; z ; a) \text { and } \phi_{p}(\beta ; \gamma ; z ; a)
$$

have the following integral representations:

$$
F_{p}(\alpha, \beta ; \gamma ; z ; a)=\frac{1}{B(\beta, \gamma-\beta)} \int_{0}^{1} t^{\beta-1}(1-t)^{\gamma-\beta-1}(1-z t)^{-\alpha} a^{\left(\frac{-p}{t(1-t)}\right)} d t
$$

for $\operatorname{Re}(\gamma)>\operatorname{Re}(\beta)>0, \operatorname{Re}(p)>0, a \in(0, \infty) \backslash\{1\}$, and $|\arg (1-z)|<\pi$.

$$
\phi_{p}(\beta ; \gamma ; z ; a)=\frac{1}{B(\beta, \gamma-\beta)} \int_{0}^{1} t^{\beta-1}(1-t)^{\gamma-\beta-1} \exp (z t) a^{\left(\frac{-p}{t(1-t)}\right)} d t,
$$

for $\operatorname{Re}(\gamma)>\operatorname{Re}(\beta)>0, \operatorname{Re}(p) \geqslant 0, a \in(0, \infty) \backslash\{1\}$,

Proof. Using relation (2.2) in equation (3.1), we have

$$
F_{p}(\alpha, \beta ; \gamma ; z ; a)=\frac{1}{B(\beta, \gamma-\beta)} \int_{0}^{1} t^{\beta-1}(1-t)^{\gamma-\beta-1} a^{\left(\frac{-p}{t(1-t)}\right)} \sum_{n=0}^{\infty}(\alpha)_{n} \frac{(z t)^{n}}{n !} d t,
$$

which on using relation (2.15) yields the desired result (3.4).

Similarly, result (3.5) can be obtained by using relation (2.2) in equation (3.2) and then using the definition of the exponential function, thus the proof of Theorem 3.3 is completed. 
Remark 3.4. Substituting $t=\frac{u}{1+u}, t=\sin ^{2} \theta$ and $t=\tan ^{2} \theta$ in relation (3.4) respectively, we get the following results:

Corollary 3.5. The modified hypergeometric function $F_{p}(\alpha, \beta ; \gamma ; z ; a)$ has the following integral representations:

$$
\begin{aligned}
& F_{p}(\alpha, \beta ; \gamma ; z ; a)=\frac{1}{B(\beta, \gamma-\beta)} \int_{0}^{1} u^{\beta-1}(1+u)^{\alpha-\gamma}(1-u(z-1))^{-\alpha} a^{\left(\frac{-p(1+u)^{2}}{u}\right)} d u, \\
& F_{p}(\alpha, \beta ; \gamma ; z ; a)=\frac{2}{B(\beta, \gamma-\beta)} \int_{0}^{\frac{\pi}{2}} \frac{\sin ^{2 b-1} \theta \cos ^{2 c-2 b-1} \theta}{\left(1-z \sin ^{2} \theta\right)^{a}} a^{\left(\frac{-p}{\sin ^{2} \theta \cos ^{2} \theta}\right)} d \theta, \\
& F_{p}(\alpha, \beta ; \gamma ; z ; a)=\frac{2}{B(\beta, \gamma-\beta)} \int_{0}^{\infty} \frac{\sinh ^{2 b-1} \theta \cosh { }^{2 a-2 c+1} \theta}{\left(\cosh ^{2} \theta-z \sinh ^{2} \theta\right)^{a}} a^{\left(\frac{-p}{\tanh ^{2} \theta \operatorname{sech}^{2} \theta}\right)} d \theta .
\end{aligned}
$$

Remark 3.6. Substituting $t=1-t$ in relation (3.5), we get the following result:

Corollary 3.7. The modified confluent hypergeometric function $\phi_{p}(\beta ; \gamma ; z ; a)$ has the following integral representation:

$$
\phi_{p}(\beta ; \gamma ; z ; a)=\frac{\exp (z)}{B(\beta, \gamma-\beta)} \int_{0}^{1} t^{\gamma-\beta-1}(1-t)^{\beta-1} \exp (-z t) a^{\left(\frac{-p}{t(1-t)}\right)} d t .
$$

Remark 3.8. Taking $z=1$ in relation (3.4) and using relation (2.2), we get the following result:

$$
F_{p}(\alpha, \beta ; \gamma ; 1 ; a)=\frac{B_{p}(\beta, \gamma-\alpha-\beta ; a)}{B(\beta, \gamma-\beta)} .
$$

Theorem 3.9. The following differentiation formulas for $\mathrm{F}_{p}(\alpha, \beta ; \gamma ; z ; \mathrm{a})$ and $\phi_{p}(\beta ; \gamma ; z ; \mathrm{a})$ hold:

$$
\begin{gathered}
\frac{\mathrm{d}^{\mathrm{k}}}{\mathrm{d} z^{\mathrm{k}}}\left\{\mathrm{F}_{\mathrm{p}}(\alpha, \beta ; \gamma ; z ; \mathrm{a})\right\}=\frac{(\alpha)_{\mathrm{k}}(\beta)_{\mathrm{k}}}{(\gamma)_{\mathrm{k}}} \mathrm{F}_{\mathrm{p}}(\alpha+\mathrm{k}, \beta+\mathrm{k} ; \gamma+\mathrm{k} ; z ; \mathrm{a}), \\
\frac{\mathrm{d}^{\mathrm{k}}}{\mathrm{d} z^{\mathrm{k}}}\left\{\phi_{\mathrm{p}}(\beta ; \gamma ; z ; \mathrm{a})\right\}=\frac{(\beta)_{\mathrm{k}}}{(\gamma)_{\mathrm{k}}} \phi_{\mathrm{p}}(\beta+\mathrm{k} ; \gamma+\mathrm{k} ; z ; \mathrm{a}) .
\end{gathered}
$$

Proof. Differentiating (3.1) with respect to $z$, we have

$$
\begin{aligned}
\frac{d}{d z}\left\{F_{p}(\alpha, \beta ; \gamma ; z ; a)\right\} & =\frac{d}{d z} \sum_{n=0}^{\infty}(\alpha)_{n} \frac{B_{p}(\beta+n, \gamma-\beta ; a)}{B(\beta, \gamma-\beta)} \frac{z^{n}}{n !} \\
& =\sum_{n=1}^{\infty}(\alpha)_{n} \frac{B_{p}(\beta+n, \gamma-\beta ; a)}{B(\beta, \gamma-\beta)} \frac{z^{n-1}}{(n-1) !} .
\end{aligned}
$$

Putting $n=n+1$ in (3.14), we have

$$
\frac{d}{d z}\left\{F_{p}(\alpha, \beta ; \gamma ; z ; a)\right\}=\sum_{n=0}^{\infty}(\alpha)_{n+1} \frac{B_{p}(\beta+n+1, \gamma-\beta ; a)}{B(\beta, \gamma-\beta)} \frac{z^{n}}{n !},
$$


which on using the following relation

$$
B(b, c-b)=\frac{c}{b} B(b+1, c-b),
$$

In the R.H.S. gives

$$
\frac{d}{d z}\left\{F_{p}(\alpha, \beta ; \gamma ; z ; a)\right\}=\frac{\alpha \beta}{\gamma} F_{p}(\alpha+1, \beta+1 ; \gamma+1 ; z ; a)
$$

Again differentiating (3.17) with respect to $z$, we obtain

$$
\frac{d^{2}}{d z^{2}}\left\{F_{p}(\alpha, \beta ; \gamma ; z ; a)\right\}=\frac{\alpha(\alpha+1) \beta(\beta+1)}{\gamma(\gamma+1)} F_{p}(\alpha+2, \beta+2 ; \gamma+2 ; z ; a)
$$

Continuing up to $\mathrm{k}$ times, we get the required result (3.12).

Applying the similar procedure used to prove result (3.12), we get the desired result (3.13), thus the proof of Theorem 3.9 is completed.

Theorem 3.10. The modified hypergeometric and confluent hypergeometric functions

$$
F_{p}(\alpha, \beta ; \gamma ; z ; a) \text { and } \phi_{p}(\beta ; \gamma ; z ; a)
$$

have the following Mellin transforms:

$$
\begin{aligned}
& \mathcal{M}\left\{\mathrm{F}_{\mathrm{p}}(\alpha, \beta ; \gamma ; z ; \mathrm{a}) ; \mathrm{p} \rightarrow \mathrm{s}\right\}=\frac{\Gamma_{\mathrm{p}}(\mathrm{s} ; \mathrm{a})}{\mathrm{B}(\beta, \gamma-\beta)} \mathrm{B}(\beta+s, \gamma-\beta+s)_{2} \mathrm{~F}_{1}(\alpha, \beta+s ; \gamma+2 s ; z), \\
& \mathcal{M}\left\{\phi_{\mathrm{p}}(\beta ; \gamma ; z ; \mathrm{a}) ; \mathrm{p} \rightarrow \mathrm{s}\right\}=\frac{\Gamma_{\mathrm{p}}(\mathrm{s} ; \mathrm{a})}{\mathrm{B}(\beta, \gamma-\beta)} \mathrm{B}(\beta+s, \gamma+\mathrm{s}-\beta) \phi(\beta+s ; \gamma+2 s ; z) .
\end{aligned}
$$

Proof. Applying Mellin transform on both sides of (3.4), we have

$$
\begin{aligned}
& \mathcal{N}\left\{F_{p}(\alpha, \beta ; \gamma ; z ; a) ; p \rightarrow s\right\} \\
= & \frac{1}{B(\beta, \gamma-\beta)} \int_{0}^{\infty} p^{s-1} \int_{0}^{1} t^{\beta-1}(1-t)^{\gamma-\beta-1}(1-z t)^{-\alpha} a^{\left(\frac{-p}{t(1-t)}\right)} d t d p,
\end{aligned}
$$

Interchanging the order of integrations in above equation, we have

$$
\begin{aligned}
& \mathcal{M}\left\{F_{p}(\alpha, \beta ; \gamma ; z ; a) ; p \rightarrow s\right\} \\
= & \frac{1}{B(\beta, \gamma-\beta)} \int_{0}^{1} t^{\beta-1}(1-t)^{\gamma-\beta-1}(1-z t)^{-\alpha}\left(\int_{0}^{\infty} p^{s-1} a^{\left(\frac{-p}{t(1-t)}\right)} d p\right) d t,(3,
\end{aligned}
$$

Using the following relations:

$$
\int_{0}^{\infty} p^{s-1} a^{\left(\frac{-p}{t(1-t)}\right)} d p=t^{s}(1-t)^{s} \Gamma_{p}(s ; a),
$$


and (2.15) in the R.H.S. of (3.21), we get

$$
\begin{aligned}
& \mathcal{M}\left\{F_{p}(\alpha, \beta ; \gamma ; z ; a) ; p \rightarrow s\right\} \\
= & \frac{\Gamma_{p}(s ; a)}{B(\beta, \gamma-\beta)} \sum_{n=0}^{\infty}(\alpha)_{n} \frac{z^{n}}{n !} \int_{0}^{1} t^{\beta+s+n-1}(1-t)^{\gamma-\beta+s-1} d t \\
= & \frac{\Gamma_{p}(s ; a)}{B(\beta, \gamma-\beta)} \sum_{n=0}^{\infty}(a)_{n} \frac{z^{n}}{n !} \frac{\Gamma(\beta+s+n) \Gamma(\gamma-\beta+s)}{\Gamma(\gamma+2 s+n)} \\
= & \frac{\Gamma_{p}(s ; a)}{B(\beta, \gamma-\beta)} B(\beta+s, \gamma-\beta+s) \sum_{n=0}^{\infty} \frac{(\alpha)_{n}(\beta+s)_{n}}{(\gamma+2 s)_{n}} \frac{z^{n}}{n !},
\end{aligned}
$$

which yields the desired result (3.19).

In similar way, we can prove result (3.20), thus the proof of Theorem 3.10 is completed.

Remark 3.11. Taking the inverse Mellin transform of both sides of equations (3.19) and (3.20), we get the following results:

$$
\begin{aligned}
\mathrm{F}_{\mathrm{p}}(\alpha, \beta ; \gamma ; z ; \mathrm{a})= & \frac{1}{2 \pi i \mathrm{~B}(\beta, \gamma-\beta)} \int_{\gamma-i \infty}^{\gamma+i \infty} \Gamma_{p}(s ; a) B(\beta+s, \gamma-\beta+s) \\
& \times{ }_{2} \mathrm{~F}_{1}(\alpha, \beta+s ; \gamma+2 s ; z) p^{-s} \mathrm{ds}
\end{aligned}
$$

and

$$
\begin{aligned}
\phi_{p}(b ; c ; z ; a)= & \frac{1}{2 \pi i B(\beta, \gamma-\beta)} \int_{\gamma-i \infty}^{\gamma+i \infty} \Gamma_{p}(s ; a) B(\beta+s, \gamma-\beta+s) \\
& \times \phi(\beta+s ; \gamma+2 s ; z) p^{-s} d s
\end{aligned}
$$

respectively.

Theorem 3.12. The modified hypergeometric and confluent hypergeometric functions

$$
F_{p}(\alpha, \beta ; \gamma ; z ; a) \text { and } \phi_{p}(\beta ; \gamma ; z ; a)
$$

have the following transformations formulas:

$$
\begin{gathered}
F_{p}(\alpha, \beta ; \gamma ; z ; a)=(1-z)^{-\alpha} F_{p}\left(\alpha, \gamma-\beta ; \gamma ; \frac{z}{z-1} ; a\right), \\
\phi_{p}(\beta ; \gamma ; z ; a)=\exp (z) \phi_{p}(\gamma-\beta ; \gamma ;-z ; a),
\end{gathered}
$$

where $|\arg (1-z)|<\pi$.

Proof. Putting $t=1-t$ in (3.4) and then using

$$
(1-z(1-t))^{-\alpha}=(1-z)^{-\alpha}\left(1+\frac{z}{1-z} t\right)^{-\alpha},
$$


in the resultant equation, we obtain

$$
F_{p}(\alpha, \beta ; \gamma ; z ; a)=\frac{(1-z)^{-\alpha}}{B(\beta, \gamma-\beta)} \int_{0}^{1} t^{\gamma-\beta-1}(1-t)^{\beta-1}\left(1+\frac{z}{1-z} t\right)^{-\alpha} a^{\left(\frac{-p}{t(1-t)}\right)} d t
$$

which in view of definition (3.1) yields the required result (3.25).

From (3.5) and (3.10), we can easily establish the required result (3.26), thus the proof of Theorem 3.12 is completed

Remark 3.13. For $a=e$, all the above relations reduce to the known results due to Chaudhry et al. [4].

\section{Conclusion}

In our present investigation, by using the idea given in reference [1], we have introduced some modified forms for basic extended special functions such as Gamma function, Beta function, hypergeometric function and confluent hypergeometric function. We have investigated some properties of these functions. Most of the special functions of mathematical physics and engineering, such as Jacobi and Laguerre polynomials can be extended by using the idea given here. Therefore, the corresponding extensions of several other familiar special functions and polynomials are need to be investigated.

\section{Acknowledgment}

The authors would like to thank the anonymous reviewer for his valuable comments and suggestions.

\section{References}

[1] Saif M, Khan F, Nisar KS and Araci S (2020). Modified Laplace transform and it is properties. J. Math. Comput. Sci. 21: 127-135.

[2] Srivastava HM and Manocha HL (1984). "A treatise on generating functions". Halsted Press, New York.

[3] Chaudhry MA, Qadir A, Rafique M and Zubair SM (1997). Extension of Euler's Beta function. J. Comput. Appl. Math. 78:19-32. https://doi.org/10.1016/S0377-0427(96)00102-1

[4] Chaudhry MA, Qadir A, Srivastava HM and Paris RB (2004). Extended Hypergeometric and Confluent Hypergeometric functions. Appl. Math. Comput. 159: 589-602. https://doi.org/10.1016/j.amc.2003.09.017

[5] Luo MJ, Milovanovic GV and Agarwal P (2014). Some results on the extended beta and extended hypergeometric functions. Appl. Math. Comput. 248: 631-651. https://doi.org/10.1016/j.amc.2014.09.110

[6] Mubeen S, Rahman G, Nisar K.S, Choi J and Arshad M (2017). An extended beta function and its properties. Far East J. Math. Sci. 102: 1545-1557.

[7] Ozergin E, Ozarslan MA and Abdullah A (2011). Extension of Gamma, Beta and Hypergeometric functions. J. Comput. Appl. Math. 235: 4601- 4610. https://doi.org/10.1016/j.cam.2010.04.019

[8] Pucheta PI (2017). A new extended Beta function. Int. J. Math. Appl. 5(3-C):255-260. http://repositorio.unne.edu.ar/handle/123456789/9105

[9] Shadab M, Jabee S and Choi J (2018). An extended beta function and its application. Far East J. Math. Sci. 103:235-251. http://dx.doi.org/10.17654/MS103010235

[10] Srivastava HM, Centinkaya A and Krymaz IO (2014). A certain generalized Pochhammer symbol and its applications to hypergeometric functions. Appl. Math. Comput. 226: 484491.https://doi.org/10.1016/j.amc.2013.10.032 
[11] Chaudhry MA and Zubair SM (1994). Generalized incomplete Gamma functions with applications. J. Comput. Appl. Math. 55: 99-124. https://doi.org/10.1016/0377-0427(94)90187-2

[12] Rainville ED (1961). "Special Functions". Macmillan Company. New York, Reprinted by Chelsea Publishing Company, Bronx, New York. 